\title{
Simplified Design Equations for a Class of Rhombic Auxetic Plates
}

\author{
Teik-Cheng Lim \\ School of Science and Technology, Singapore University of Social Sciences, Singapore
}

\begin{abstract}
Equations for solving the deflection and bending moments of rhombic plates by exact method are known to be highly tedious. A set of simplified equations is developed for design purposes of such simply supported plates under uniform load. Curve-fitting from exact data allows the deflection and its second derivatives, evaluated at the plate centre, to be expressed in greatly simplified and yet sufficiently accurate empirical models for thin rhombic plates. Using the simplified model, it is shown that the maximum bending moment can be reduced by using auxetic materials. By including the effects of shear deformation for thick rhombic plates, it is demonstrated that the ratio of shear-to-bending deformation decreases as the rhombic plate approaches a square shape and as the plate's Poisson's ratio becomes more negative.
\end{abstract}

\section{Introduction}

The Poisson's ratio of materials is normally assumed to be positive, with a value of $v=0.3$ being typically assigned for isotropic materials. Although the Poisson's ratio of anisotropic solids has no bounds [1], it has been shown that the Poisson's ratio in the case of isotropic materials are bounded within $-1 \leq v \leq 0.5$ [2]; solids with negative Poisson's ratio have been studied [3]. A number of auxetic materials applications have been suggested; interested readers are referred to related reviews [4-6] and a monograph [7] for further details of such materials. Investigations into the performance of plates with the use of auxetic materials have been carried out [8-11]. Although studies on auxetic plates have been done on rectangular [12-14], circular [15-17], triangular [18-20], elliptical [21], sectorial [22], hexagonal [23] and regular polygonal [24] plates-no work has been done on auxetic rhombic plates. This paper aims to provide a set of design equations that is simple to execute and sufficiently accurate for a class of rhombic plates, which are simply supported and uniformly loaded, with special emphasis on the use of auxetic materials.

\section{Preamble to thin rhombic plates}

With reference to Fig.1 for a simply-supported rhombic plate of thickness $h$, sides $a$ and complementary angles of $2 \alpha$ and $(\pi-2 \alpha)$ under uniformly distributed load of $q_{0}$, the deflection $w^{K}$ of such plate within the context of Kirchhoff plate theory

$$
w^{K}=w_{0}+w_{1}
$$

at any point $(r, \theta)$ is given by the summation of a particular solution $w_{0}$

$$
w_{0}=\frac{q_{0}}{D} \frac{r^{4}}{64}\left(1-\frac{4 \cos 2 \theta}{3 \cos 2 \alpha}+\frac{\cos 4 \theta}{3 \cos 4 \alpha}\right)
$$

and a complementary function $w_{1}$

$$
\begin{aligned}
& w_{1}=\frac{q_{0}}{D} \sum_{m=1}^{\infty}\left(a_{m}+b_{m} r^{2}\right) r^{\lambda_{m}+1} \cos \left(\lambda_{m}+1\right) \theta \\
& w_{1}=\frac{q_{0}}{D} \sum_{n=1}^{\infty}\left(a_{n}+b_{n} r^{2}\right) r^{\lambda_{n}} \cos \lambda_{n} \theta
\end{aligned}
$$

according to Morley's [25] or Warren's nomenclature, respectively, where

$$
\begin{aligned}
& \lambda_{m}=\frac{(2 m-1) \pi}{2 \alpha}-1 \\
& \lambda_{n}=\frac{(2 n-1) \pi}{2 \alpha}
\end{aligned}
$$

while the plate's flexural rigidity is

$$
D=\frac{E h^{3}}{12\left(1-v^{2}\right)}
$$

in which $E$ is the Young's modulus and $v$ is the Poisson's ratio of the plate material. One may note that the plate's Poisson's ratio in Eqs.(1) and (2) are manifested only through the flexural rigidity.

The methods for evaluating the coefficients $b_{m}$ and $a_{m}$ by Morley [25] and $b_{n}$ and $a_{n}$ by Warren [26] are tedious, and are summarized in the Appendices A1 and A2, respectively. Warren [26] appreciated the fact that the coefficients $b_{m}$ and $a_{m}$ are chosen by Morley [25] so as to minimize the integral square error over $\mathrm{AB}$. This leads to numerous integrals which are tedious to evaluate, and then finally to the solution of $M$ by $M$ simultaneous linear equations for determining these 
coefficients. Warren [26] proposed the method of "point matching" that requires satisfying Eqs.(A11) and (A12) exactly at $M$ discrete points along $\mathrm{AB}$, which leads directly to two sets of $M$ by $M$ simultaneous linear equations for determining the said coefficients. Both ways are nevertheless tedious to the designer, and a simplified model would be of great practical importance. To provide simplified design equations for thin rhombic plates, results from Morley [25] are used for generating semi-empirical models that are very convenient and yet sufficiently accurate. Thereafter, the simplified design equations for thin plates form the basis for evaluating the shear deformation in thick rhombic plates.

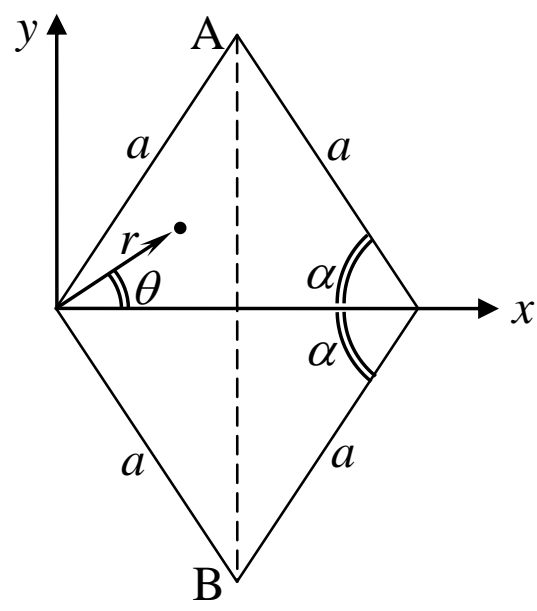

Figure 1. Schematic diagram of a rhombic plate.

\section{Semi-empirical modelling for thin rhombic plates}

Perusal to Eqs.(1) to (4), as well as Appendices A1 and A2, shows that the plate deflection is a function of load $q_{0}$, location $(r, \theta)$, half angle of obtuse corner $\alpha$ as well as the Young's modulus, plate thickness and Poisson's ratio. If one were to express the plate deflection in the form of $w D /\left(q_{0} a^{4}\right)$, then this dimensionless plate deflection is independent from the material properties $(E, v)$ of the plate. Based on Morley's [47] numerical results for the dimensionless deflection as well as the bending moments at the center of the plate for $v=0.3$ and $45^{\circ} \leq \alpha \leq 75^{\circ}$, a curve-fitting exercise yields the following semi-empirical models for the plate center:

$$
\begin{aligned}
& \frac{w_{\max }^{K} D}{q_{0} a^{4}}=\left\{\begin{array}{llll}
1.907 & 3.578 & 2.069 & 3.397
\end{array}\right\}\left\{\begin{array}{l}
+10^{-7} \alpha^{3} \\
-10^{-5} \alpha^{2} \\
+10^{-3} \alpha^{1} \\
-10^{-2} \alpha^{0}
\end{array}\right\} \\
& \frac{D}{q_{0} a^{2}}\left\{\begin{array}{l}
\frac{\partial^{2} w_{\max }^{K}}{\partial x^{2}} \\
\frac{\partial^{2} w_{\max }^{K}}{\partial y^{2}}
\end{array}\right\}=\left[\begin{array}{ccc}
3.768 & 3.861 & 6.039 \\
0 & -1.071 & -8.604
\end{array}\right]\left\{\begin{array}{l}
-10^{-5} \alpha^{2} \\
+10^{-3} \alpha^{1} \\
-10^{-2} \alpha^{0}
\end{array}\right\}
\end{aligned}
$$

where $\alpha$ in the semi-empirical models are expressed in degrees. Since Eqs.(1) to (3) are independent from the plate's Poisson's ratio when non-dimensionalized, it follows that this independence holds for Eqs.(6) and (7). Nevertheless the Poisson's ratio plays its role in the bending moment, as described in Eq.(8).
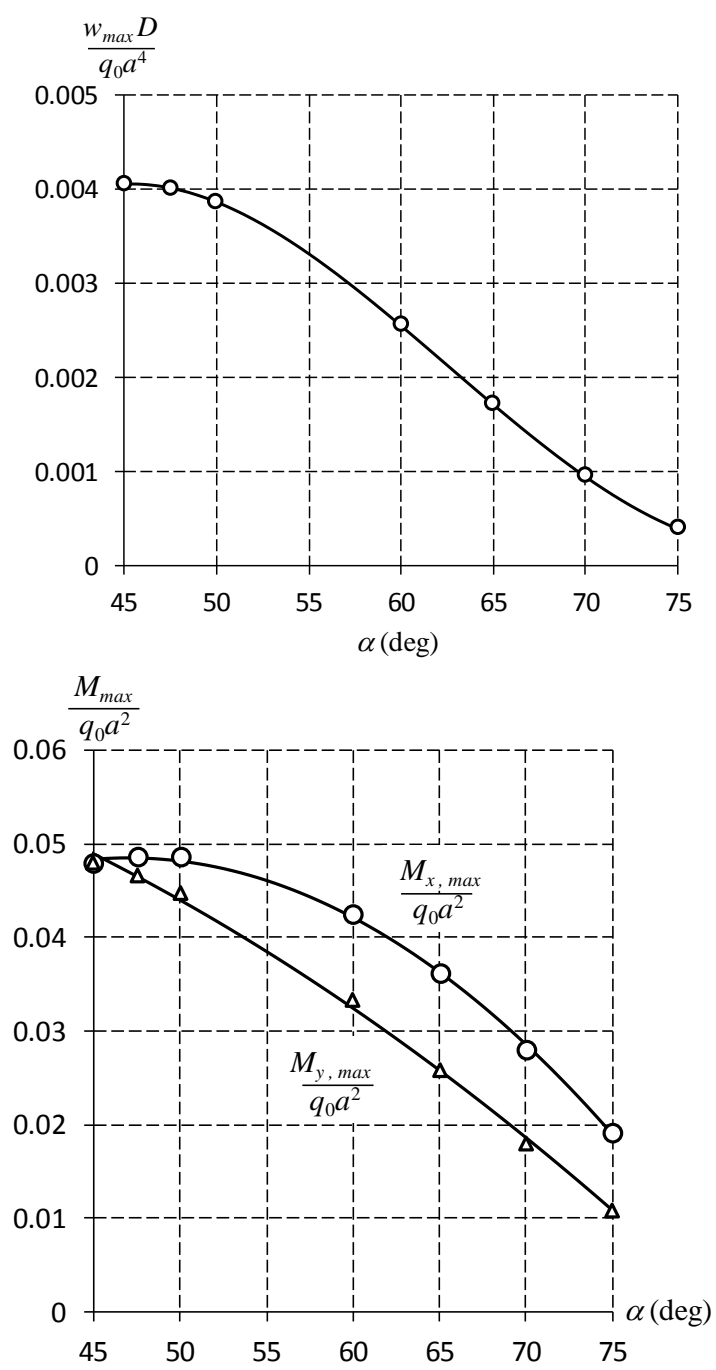

Figure 2. Comparison between the semi-empirical (smooth curves) and Morley's [47] exact numerical results (data points) for the dimensionless maximum deflection (top) and the dimensionless maximum bending moments along the $x$ - and $y$ axes (bottom) for $v=0.3$ and $45^{\circ} \leq \alpha \leq 75^{\circ}$.

Verification of the semi-empirical maximum deflection model described in Eq.(6) is made by plotting it in comparison to the discrete data points by Morley, as shown in Fig.2 (top). Apart from the maximum deflection, another plate response that is of interest to the designer is the maximum stresses. Since the bending stress is related to the bending moment, i.e. $\sigma_{\max }=6 M_{\max } / h^{2}$, it suffices to evaluate the maximum bending moments. Substitution of Eq.(7) and $v=0.3$ into

$\left\{\begin{array}{l}M_{x, \max } \\ M_{y, \max }\end{array}\right\}=-D\left[\begin{array}{ll}1 & v \\ v & 1\end{array}\right]\left\{\begin{array}{l}\frac{\partial^{2} w_{\max }^{K}}{\partial x^{2}} \\ \frac{\partial^{2} w_{\max }^{K}}{\partial y^{2}}\end{array}\right\}$ 
allows the semi-empirical bending moments to be compared with the exact results, as plotted in Fig.2 (bottom), in which $M_{\max }=M_{x, \max }>M_{y, \text { max }}$ for $\alpha>45^{\circ}$. Having established their validity, the semi-empirical models are used for plotting the loci of dimensionless maximum bending moments in the conventional $0 \leq v \leq 0.5$, moderately auxetic $-0.5 \leq v<0$ and highly auxetic $-1 \leq v<-0.5$ regions. Reference to Fig. 3 shows that, with all other parameters fixed, the maximum bending moment-and hence the maximum bending stress-is reduced by the extent of material auxeticity.

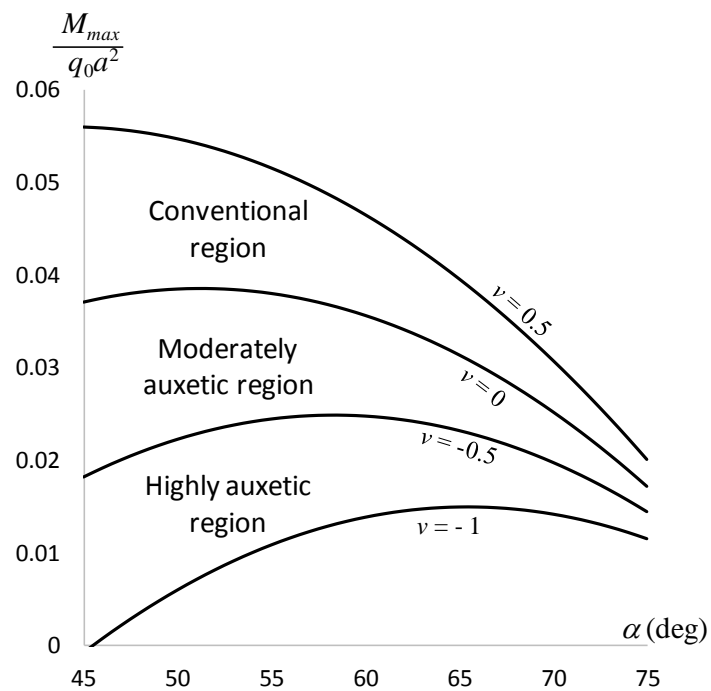

Figure 3. Loci of dimensionless maximum bending stress for conventional, moderately auxetic and highly auxetic regions.

\section{First-order shear model for thick rhombic plates}

The use of Kirchhoff plate theory, which accounts for the plate deflection on the basis of plate bending, in the preceding section is valid for thin plates. Thick plates experience pronounced transverse shear deformation. This section attempts to evaluate the shear deformation in thick rhombic plates using first-order shear deformation theory (FSDT). The FSDT by Mindlin [27] assumes constant out-of-plane shear strain, thereby incurring constant out-of-plane shear stress, across the plate's thickness. For this reason, a shear correction factor $\kappa$ is required to correct the shear modulus $G$; the Mindlin plate deflection $w^{M}$ has been shown to be related to the Kirchhoff plate deflection $w^{M}$ as [28]

$$
w^{M}=w^{K}+\frac{\mathrm{M}}{\kappa G h}
$$

where the Marcus moment of the Kirchhoff plate is

$$
\mathrm{M}=-D \nabla^{2} w^{K} \text {. }
$$

The deflection relationship between the Kirchhoff and Mindlin plate theories described in Eq.(9) can be expressed in a more tractable form as (e.g. $[23,24])$

$$
w^{M}=w^{K}-\frac{h^{2}}{6 \kappa(1-v)} \nabla^{2} w^{K} .
$$

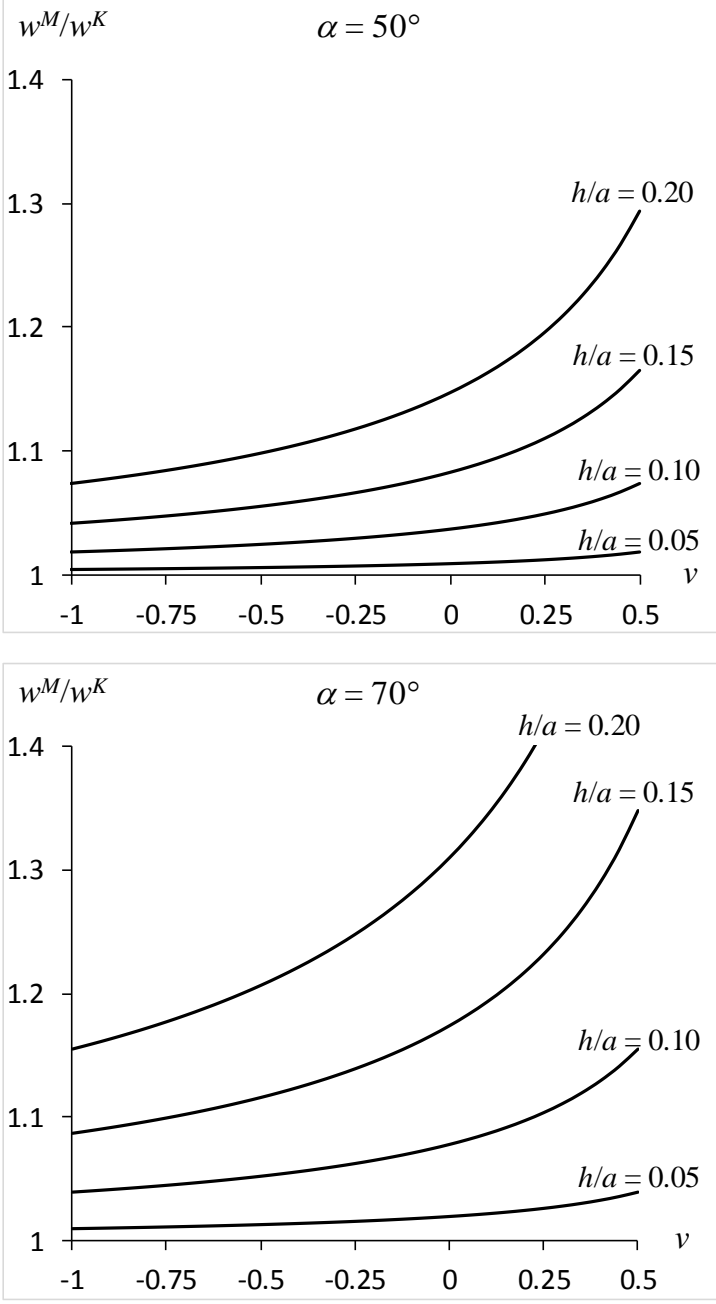

Figure 4. Plots of Mindlin-to-Kirchhoff plate deflection ratio versus Poisson's ratio at various relative plate thickness for $\alpha=50^{\circ}$ (top) and $\alpha=70^{\circ}$ (bottom).

From the semi-empirical models of Eqs.(6) and (7), as well as the shear correction factor $\kappa=5 / 6$, the ratio of Mindlin-to-Kirchhoff plate deflection ratio at the plate center can be obtained as

$\frac{w_{\max }^{M}}{w_{\max }^{K}}=1-\frac{h^{2} / a^{2}}{5(1-v)} \frac{f(\alpha)}{g(\alpha)}$

where

$f(\alpha)=3.768 \times 10^{-5} \alpha^{2}-2.79 \times 10^{-3} \alpha-0.02565$

and

$$
\begin{aligned}
g(\alpha)= & 1.907 \times 10^{-7} \alpha^{3}-3.578 \times 10^{-5} \alpha^{2} \\
& +2.069 \times 10^{-3} \alpha-0.03397
\end{aligned}
$$

Figures 4 and 5 furnish plots of Mindlin-to-Kirchhoff plate deflection ratio at the plate center for moderately sharp $\left(\alpha=50^{\circ}\right)$ and significantly sharp $\left(\alpha=70^{\circ}\right)$ rhombic plates for various relative thickness $h / a$ and Poisson's ratio $v$. A full range of $w^{M} / w^{K}$ plotted with respect to the rhombic plate's half angle $\alpha$ for various Poisson's ratio at $h / a=0.2$ is given in Fig.6. 


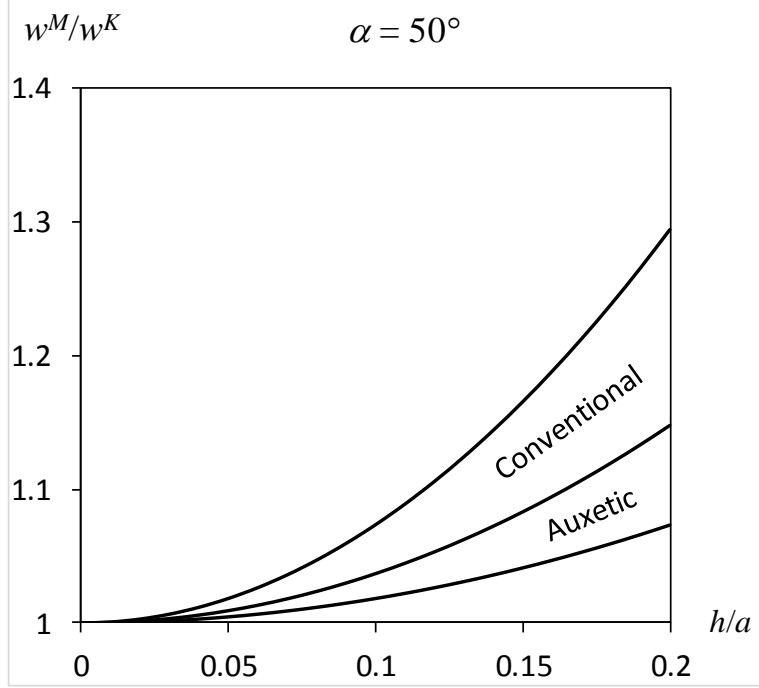

$w^{M / w^{K}} \quad \alpha=70^{\circ}$

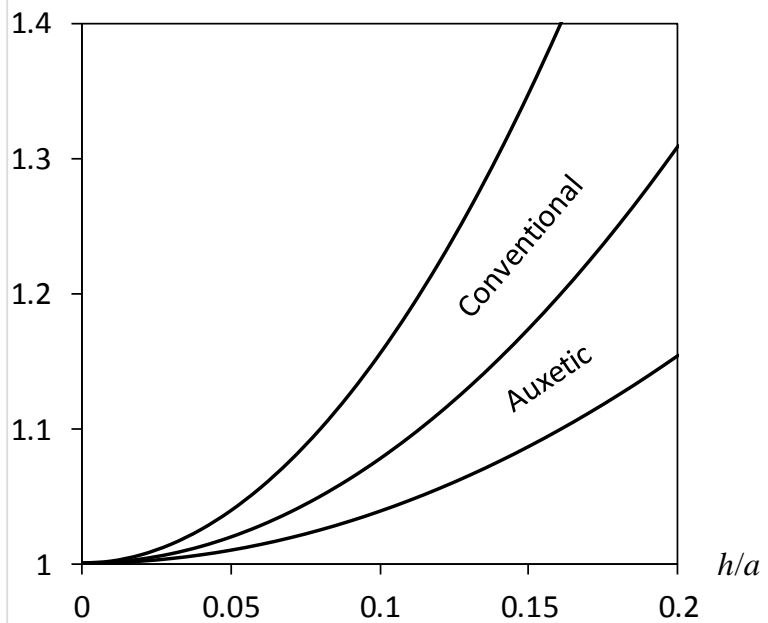

Figure 5. Demarcation of auxetic region from conventional region at $\alpha=50^{\circ}$ (top) and $\alpha=70^{\circ}$ (bottom).

As expected, the extent of shear deformation as a ratio of bending deformation diminishes with decreasing plate thickness; more interestingly, the shear-to-bending ratio decreases (a) as the plate Poisson's ratio becomes more negative, and (b) as the plate approaches a square, i.e. $\alpha \rightarrow 45^{\circ}$.

\section{Conclusions and recommendations}

A set of simplified design equations for describing the deflection and second derivatives at the centre of a simply-supported uniformly-loaded thin rhombic plates has been developed herein by back calculation from numerical results based on exact model in dimensionless forms, which are independent from the Poisson's ratio.

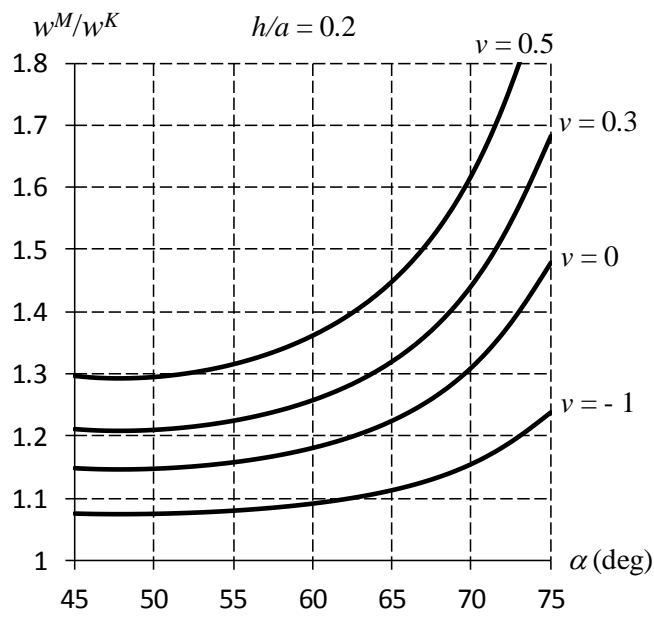

Figure 6. A family of $w^{M} / w^{K}$ curves plotted against the half angles $\alpha$ of rhombic plates at dimensionless thickness of $h / a=0.2$.

Results from the thin plate theory reveals that auxeticity decreases the dimensionless maximum bending moments, and hence the maximum bending stress, of thin rhombic plates. Extending this thin plate model to thick rhombic plates, it was found that the ratio of shear deformation vis-à-vis bending deformation increases with the dimensionless plate thickness $h / a$, half angle $\alpha$, and Poisson's ratio. These observations suggest that the analysis of thick rhombic plates can be further simplified to thin plate theory if the thick rhombic plates are more regular and/or made from auxetic materials.

\section{References}

1. T.C.T. Ting, T. Chen, Quart. J. Mech. Appl. Math. 58, 73 (2005)

2. L.D. Landau, E.M. Lifshitz, Theory of Elasticity, Pergamon Press, Oxford (1970)

3. K.W. Wojciechowski, Mol. Phys. 61, 1247 (1987)

4. K.K. Saxena, R. Das, E.P. Calius, Adv. Eng. Mater. 18, 1847 (2016)

5. T.C. Lim, Phys. Status Solidi RRL 11, 1600440 (2017)

6. R.S. Lakes, Ann. Rev. Mater. Res. 47, 63 (2017)

7. T.C. Lim, Auxetic Materials and Structures, Springer, Singapore (2015)

8. T.C. Lim J. Eng. Mech. 136, 1176 (2010)

9. T.C. Lim, Smart Mater. Struct 25, 054008 (2016)

10. P. Sobieszczyk, M. Majka, D. Kuźma, T.C. Lim, P. Zieliński, Phys. Status Solidi B 252, 1615 (2015)

11. L. Boldrin, S. Hummel, F. Scarpa, D. Di Maio, C. Lira, M. Ruzzene, C.D.L. Remillat, T.C. Lim, R. Rajasekaran, S. Patsias, Compos. Struct. 149, 114 (2016)

12. T.C. Lim, J. Mater.: Des. Appl. 227, 111 (2013)

13. T.C. Lim, J. Eng. Mater. Technol. 136, 031007 (2014)

14. T.C. Lim, Int. J. Mech. Mater. Eng. 11, 13 (2016)

15. T.C. Lim, J. Mech. 29, 121 (2013)

16. T.C. Lim, 2014 J. Eng. Mater. Technol. 136, 021007 (2014) 
17. T.C. Lim, J. Eng. Mater. Technol. 138, 041011 (2016)

18. T.C. Lim, Mech. Based Des. Struct. Mach. 44, 514 (2016)

19. T.C. Lim, J. Eng. Mater. Technol. 138, 014501 (2016)

20. T.C. Lim, Smart Mater. Struct. 25, 054001 (2016)

21. T.C. Lim, J. Mech. 32, 413 (2016)

22. T.C. Lim, Phys. Status Solidi B 254, 1600784 (2017)

23. T.C. Lim, Phys. Status Solidi B 254, 1700014 (2017)

24. T.C. Lim, Proc. Inst. Civil Eng. - Eng. Comput. Mech. 170, 167 (2017)

25. L.S.D. Morley, Quart. J. Mech. Appl. Math. 15, 413 (1962)

26. W.E. Warren, AIAA J. 2, 166 (1964)

27. R.D. Mindlin, J. Appl. Mech. 18, 31 (1951)

28. C.M. Wang, W.A.M. Alwis, J. Eng. Mech. 121, 1383 (1995).

\section{Appendix A1: Evaluation of coefficients $b_{n}$ and $a_{n}$ by Morley (1962)}

The calculations leading to the solutions of coefficients $b_{n}$ requires

$\delta \int_{-a \sin \alpha}^{a \sin \alpha}\left[\frac{\partial}{\partial x} \nabla^{2} w^{K}\right]_{x=a \cos \alpha}^{2} d y=0$

that leads to a system of $M$ equations

$$
\left[\begin{array}{cccc}
A_{11} & A_{12} & \cdots & A_{1 M} \\
A_{21} & A_{22} & & \\
\vdots & & \ddots & \\
A_{M 1} & & & A_{M M}
\end{array}\right]\left\{\begin{array}{c}
b_{1} \\
b_{2} \\
\vdots \\
b_{M}
\end{array}\right\}=\left\{\begin{array}{c}
A_{1} \\
A_{2} \\
\vdots \\
A_{M}
\end{array}\right\}
$$

where

$A_{m n}=A_{n m}=16\left(\lambda_{m}+2\right)\left(\lambda_{m}+1\right)\left(\lambda_{n}+2\right)\left(\lambda_{n}+1\right)$

$\times \int_{-a \sin \alpha}^{a \sin \alpha}\left[r^{\lambda_{m}+\lambda_{n}} \cos \lambda_{m} \theta \cos \lambda_{n} \theta\right]_{x=a \cos \alpha} d y$

and

$\frac{A_{m}}{4\left(\lambda_{m}+2\right)\left(\lambda_{m}+1\right)}$

$=\frac{1}{2}\left(\frac{1}{\cos 2 \alpha}-1\right)_{-a \sin \alpha}^{a \sin \alpha}\left[r^{\lambda_{m}+1} \cos \lambda_{m} \theta \cos \theta\right]_{x=a \cos \alpha} d y$

$=\frac{(-1)^{m+1}(1-\cos 2 \alpha) a^{\lambda_{m}+2}}{\lambda_{m}+1}$

while calculations leading to the solutions of coefficients $a_{n}$ requires

$\delta \int_{-a \sin \alpha}^{a \sin \alpha}\left[\frac{\partial w^{K}}{\partial x}\right]_{x=a \cos \alpha}^{2} d y=0$

that leads to another system of $M$ equations

$\left[\begin{array}{cccc}A_{11}{ }^{\prime} & A_{12}{ }^{\prime} & \cdots & A_{1 M}{ }^{\prime} \\ A_{21}{ }^{\prime} & A_{22}{ }^{\prime} & & \\ \vdots & & \ddots & \\ A_{M 1}{ }^{\prime} & & & A_{M M}{ }^{\prime}\end{array}\right]\left\{\begin{array}{c}a_{1} \\ a_{2} \\ \vdots \\ a_{M}\end{array}\right\}=\left\{\begin{array}{c}A_{1}{ }^{\prime} \\ A_{2}{ }^{\prime} \\ \vdots \\ A_{M}{ }^{\prime}\end{array}\right\}$
$A_{m n}{ }^{\prime}=A_{n m}{ }^{\prime}=\frac{A_{m n}}{16\left(\lambda_{m}+2\right)\left(\lambda_{n}+2\right)}$

and

$$
A_{m}{ }^{\prime}=\left(\lambda_{m}+1\right) \int_{-a \sin \alpha}^{a \sin \alpha}\left[\chi r^{\lambda_{m}} \cos \lambda_{m} \theta\right]_{x=a \cos \alpha} d y
$$

in which $A_{m n}$ is given by Eqs.(A3) while

$$
\begin{aligned}
\chi & =\frac{r^{3}}{16}\left[\left(1-\frac{1}{\cos 2 \alpha}\right) \cos \theta+\frac{1}{3}\left(\frac{1}{\cos 4 \alpha}-\frac{1}{\cos 2 \alpha}\right) \cos 3 \theta\right] \\
& +\sum_{m=1}^{M} b_{m}\left\{\left(\lambda_{m}+2\right) \cos \lambda_{m} \theta+\cos \left(\lambda_{m}+2\right) \theta\right\} r^{\lambda_{m}+2}
\end{aligned}
$$

\section{Appendix A2: Evaluation of coefficients $b_{n}$ and $a_{n}$ by Warren (1964)}

The coefficients $a_{n}$ and $b_{n}$ are chosen ideally so as to satisfy symmetry boundary conditions along the diagonal $\mathrm{AB}$ (See Fig.1). These conditions require

$$
\left.\frac{\partial}{\partial x} \nabla^{2} w^{K}\right|_{\mathrm{AB}}=0 \quad,\left.\quad \frac{\partial w^{K}}{\partial x}\right|_{\mathrm{AB}}=0
$$

which give

$\left\{a \cos \alpha\left(1-\frac{1}{\cos 2 \alpha}\right)+8 \sum_{n=1}^{\infty} \lambda_{n}\left(\lambda_{n}+1\right) b_{n} r^{\lambda_{n}-1} \cos \left(\lambda_{n}-1\right) \theta\right\}_{\mathrm{AB}}$

$=0$

and

$\left\{\frac{r^{3}}{16}\left[\left(1-\frac{1}{\cos 2 \alpha}\right) \cos \theta+\frac{1}{3}\left(\frac{1}{\cos 4 \alpha}-\frac{1}{\cos 2 \alpha}\right) \cos 3 \theta\right]\right.$

$+\sum_{n=1}^{\infty} \lambda_{n} a_{n} r^{\lambda_{n}-1} \cos \left(\lambda_{n}-1\right) \theta$

$\left.+\sum_{n=1}^{\infty} b_{n} r^{\lambda_{n}+1}\left[\left(\lambda_{n}+1\right) \cos \left(\lambda_{n}-1\right) \theta+\cos \left(\lambda_{n}+1\right) \theta\right]\right\}_{\mathrm{AB}}=0$

where 